\title{
Una scheda di valutazione delle competenze del personale: strumento per il coordinatore infermieristico dei servizi di dialisi
}

\author{
L. Napoli \\ C.A.L. di Dialisi, Settimo Torinese e U.O. Nefrologia e Dialisi ASL TO4, Chivasso (Torino)
}

EVALUATION REPORT OF HEMODIALYSIS STAFF EXPERTISE: DEVICE FOR HEAD NURSE IN HEMODIALYSIS UNITS

ABSTRACT. Health Care workers competence validation is a strategic factor for National Healthcare System excellence; for Nurse Unit Manager it supports care planning responsibility. A Competence Validation Scheme (SVC) has been implemented as possible tool for objective nurses' competence evaluation. The SVC could be used either as continuous self evaluation tool or for monitoring a new nurse level of training and knowledge. The SVC defines different areas of clinical practice and different level of competence to task performance, using a Likert scale. The SVC has been sent to 26 dialysis centers in Piemonte and Valle d'Aosta Regions, using a Delphy system of inquiry. A questionnaire addressed to nurse unit managers had been sent in parallel, in order to investigate the process of competence evaluation in their unit. The $38,5 \%$ of units provided feed-back. The SVC had been tested in 20 nurses, too. None expressed negative comments: the SVC has been considered a potential specific, broad and useful tool to allow self evaluation. A larger pair testing is need to effective tool validation.

KEY WoRDS. Self-evaluation, Competence, Evaluation

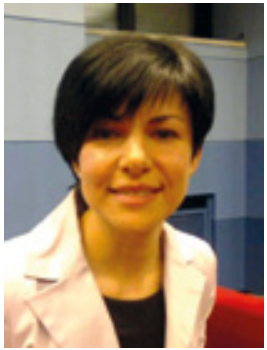

Luisa Napoli

\section{Introduzione}

I concetti di autonomia e responsabilità (1), ben si legano al significato del 'valutare' e all'importanza che la valutazione assume nella gestione delle risorse umane. La valutazione delle performance diventa in tale ottica una tematica cruciale negli attuali contesti organizzativi, dove la risorsa umana si distingue attraverso la capacità degli individui di acquisire, governare e applicare il patrimonio di conoscenze (2). Nel nostro ordinamento giuridico e professionale non è presente alcun concetto di sistematicità e periodicità nella valutazione del professionista il quale, quindi, mantiene per tutta la vita lavorativa la condizione di 'esperto' dell'area di competenza e di esclusività professionale. La valutazione delle competenze dei professionisti rimane un fattore strategico per l'eccellenza del sistema sanitario nazionale (3) e per tale ragione nel nostro Paese la normativa è ricca di leggi e di accordi necessari a definire un sistema nazionale di certificazione delle competenze nella formazione professionale (4). In qualità di futuro Coordinatore Infermieristico (C.I.) è stato significativo progettare, attraverso la partecipazione attiva nel gruppo infermieristico della Dialisi del Presidio Ospedaliero San Giovanni Bosco di Torino un lavoro con risvolti pratici come declinare una Scheda di Valutazione delle Competenze (5) (SVC). La SVC ha trovato origine dalla formulazione precedente (obiet- 
tivo aziendale 2009) di un Profilo delle Competenze e ha rappresentato da un lato l'impegno di elaborare uno strumento riconosciuto e validato all'interno del contesto dialitico utile a stimolare nell'infermiere l'autovalutazione e l'autoriflessione e dall'altro guida per il C.I. alla valutazione oggettiva attraverso un colloquio reciprocamente costruttivo.

\section{Materiali e Metodi}

È stata condotta una ricerca bibliografica attraverso la consultazione di banche dati quali CINHAL e MEDLI$\mathrm{NE}$ con l'obiettivo di conoscere le caratteristiche del processo di valutazione o la classificazione delle competenze dell'infermiere di nefrologia e dialisi fuori dal nostro Paese. Il risultato è stato che, in tale contesto, il professionista raggiunge una preparazione di alto livello con specializzazioni differenziate per ramo d'impiego (terapia intensiva nefrologica, nefrologia e terapia sostitutiva), seguendo corsi specifici e superando un esame di certificazione (6-8). In un sistema così organizzato è stato difficile trovare dei punti comuni con la realtà italiana nella quale la preparazione utile a supportare la cronicità di un paziente così complesso è quantomeno carente. Il professionista impara la gestione tecnico-relazionale negli anni, direttamente nel contesto e non senza difficoltà o errori e per tale ragione la valutazione continua assume importanti risvolti. Perseguendo l'obiettivo sopra citato sono stati allargati i termini della ricerca a contesti non dialitici; si è evidenziato l'utilizzo di un approccio metodologico comune così strutturato: iniziale identificazione delle competenze specifiche per il ruolo occupato (9-16) e costruzione di schede con scale di misurazione tipo Likert delle competenze stesse declinate in attività (17). La competenza (18), elemento composto da capacità, conoscenze, ed esperienze finalizzate non è osservabile e quantificabile di per se stessa, diventa necessario scomporla in elementi di attività sulla base del ruolo che il professionista ricopre. Parallelamente a questa ricerca si è stilato un Profilo delle Competenze, strumento che per la sua duttilità, e non ultima la caratteristica di sostenere il cambiamento autodiretto, poteva dimostrarsi utile come base della SVC. Gli 86 elementi di attività individuati nel Profilo di Posto (distinti da un numero progressivo per una rapida identificazione) suddivisi in 4 funzioni e in 13 sottoaree sono diventati i punti sui quali formulare la valutazione del Professionista. Si è ricorsi ad organizzare lo strumento in gradi e livelli per associare al valutato un punteggio finale che rendesse più immediato il grado di performance raggiunto. I primi due gradi sono caratterizzati ciascuno da tre livelli, il terzo, nonché, ultimo grado, si identifica in un unico livello il settimo. A ogni elemento di attività corrisponde il punteggio del livello di riferimento (da 1 a 7); è previsto il raggiungimento di un punteggio minimo che cambia il suo valore a seconda che si tratti di un infermiere neoassunto (258 punti), o di uno che lavora da almeno un anno (430 punti). Il punteggio massimo è rappresentato dal raggiungimento del livello 7 per tutte le attività elencate (602 punti). Il primo modello di SVC è nato per la valutazione dell'infermiere di emodialisi, ma a strumento perfezionato, si potranno individuare le competenze specifiche dell'infermiere di Dialisi Peritoneale e di Ambulatorio Nefrologico e differenziando le schede attraverso un codice colore oltre che una diversa dicitura iniziale. L'utilizzo della SVC prevede un'attenta lettura della legenda posta sul frontespizio sia da parte del valutatore, sia da parte del valutato e, poiché è auspicabile che il professionista a seguito di un'autoanalisi si autovaluti (19), è indispensabile che lo strumento gli venga consegnato con sufficiente anticipo rispetto al colloquio con il proprio C.I. Momento nel quale le due figure di professionisti si impegnano ad affrontare in modo costruttivo le eventuali discrepanze e criticità presenti nella valutazione. Il passo successivo alla costruzione della SVC è stato quello di testarla. A tale scopo, attraverso un indagine 'Delphi' (20), si è costituito un gruppo di esperti ai quali fornire lo strumento per una prima valutazione e integrazione (primo round-fase esplorativa). Il panel è stato individuato nelle figure dei C.I. appartenenti a 26 Centri Dialisi di Piemonte e Valle d'Aosta, ognuno di loro ha ricevuto tramite e-mail o fax la SVC, gli è stato chiesto di analizzarla, formulare una valutazione dello strumento in qualità di C.I. e testarla in forma anonima anche solo con un infermiere per Centro e formulando, a questo punto, un secondo giudizio sulla validità dello strumento ai fini dell'autovalutazione e dell'autoriflessione in qualità di infermiere. L'indagine strutturata in più round (il feedback, l'analisi dei risultati), come previsto dal metodo, ha determinato la presentazione dello strumento con le modifiche apportate e i relativi questionari utili al proseguimento dell'indagine fino al chiarimento dei termini di disaccordo o il raggiungimento di una convergenza ritenuta soddisfacente all'interno del gruppo di partecipanti. L'intero processo di indagine si è prefissato, attraverso la creatività e la competenza dell'intero panel di esperti, di validare lo strumento di partenza apportando le necessarie modifiche. Contemporaneamente al testing a ogni C.I. è stato somministrato un breve questionario per indagare le modalità di valutazione in uso nel contesto di appartenenza e l'esistenza o meno di una scheda di valutazione codificata. 


\section{Risultati}

A seguire il questionario presentato ai C.I. dei 26 Centri Dialisi di Piemonte e Valle d'Aosta. Solo 10 rispondono, il $38.5 \%$ dei vari Centri e riporta il totale dei dati raccolti:

\begin{tabular}{|l|l|l|}
\hline & SI & NO \\
\hline $\begin{array}{l}\text { 1) Presso il Servizio Dialisi da Lei coordinato avviene } \\
\text { la valutazione continua del Personale? }\end{array}$ & 6 & 4 \\
\hline 2) La cadenza con cui avviene è annuale? & 4 & \\
\hline 3) È semestrale? & 1 & 1 non ha precisato \\
\hline 4) Si avvale di uno strumento ? & 5 & 1 \\
\hline
\end{tabular}

\begin{tabular}{|l|l|l|l|}
\hline & $\begin{array}{l}\text { Scheda valutativa / } \\
\text { Check-list basata su } \\
\text { valori numerici tipo } \\
\text { scale (Likert ecc. })\end{array}$ & $\begin{array}{l}\text { Scheda con } \\
\text { possibilità di } \\
\text { esprimere valutazioni } \\
\text { "aperte / discorsive” }\end{array}$ & Altro \\
\hline $\begin{array}{l}\text { 5) Lo strumento in } \\
\text { questione è : }\end{array}$ & $\begin{array}{l}4(+2 \text { anche } \\
\text { discorsivo) }\end{array}$ & 1 & 1 \\
\hline
\end{tabular}

6) Se ha risposto "ALTRO" alla precedente domanda può brevemente spiegare di che strumento si tratta ? Può cortesemente allegarlo al questionario e inviarlo al fax di riferimento?.....

\begin{tabular}{|l|l|l|}
\hline & SI & NO \\
\hline 7) Lo strumento utilizzato è specifico per il Suo settore? & 1 & 5 \\
\hline $\begin{array}{l}\text { 8) Si tratta di uno strumento unificato in uso a tutta } \\
\text { l'Azienda? }\end{array}$ & 5 & \\
\hline $\begin{array}{l}\text { 9) Risponde alle Sue esigenze e a quelle del } \\
\text { Personale nel predisporre una corretta valutazione }\end{array}$ & 1 & 1 \\
e unasoddisfacente autovalutazione? & 2 & 2 \\
\hline
\end{tabular}

\section{Discussione}

La scarsa adesione all'indagine da parte dei Centri Dialisi contattati (risponde solo il $38.5 \%$ ), restituisce una fotografia sfocata della realtà dialitica piemontese. I Centri aderenti all'indagine risultano tutti diretti da C.I. provvisti di titolo riconosciuto. L'anzianità media di coordinamento nel settore è di 12 anni, con un minimo di 5 anni e un massimo di 32 . Indagando l'esistenza di un processo valutativo continuo degli Infermieri: 4 Centri hanno dichiarato che non eseguono la valutazione; dove avviene, la frequenza è annuale per 4 Centri e semestra- le per 1. Metà dei Centri si avvale di uno strumento, per 4 si tratta di una scheda tipo check-list basata su valori numerici, 2 sul loro strumento possono aggiungere commenti e valutazioni discorsive, 1 utilizza una scheda di valutazione sulla quale il giudizio è espresso in modo discorsivo, 1 Centro esprime la valutazione avvalendosi dell'osservazione diretta e un colloquio tra C.I. e Professionista. Sulla specificità degli strumenti valutativi per il settore dialitico 1 solo Centro ha confermato di possederne uno. 5 utilizzano uno strumento unificato per tutta l'azienda. Due C.I. hanno dichiarato che il loro strumen- 
to soddisfa solo parzialmente le esigenze di valutazione poiché si tratta, in un caso, di una scheda concepita per la valutazione del CPSE appartenente all'area critica e, nell'altro caso, di uno strumento che favorisce l'approfondimento delle aree relazionale ed emozionale.

L'opinione comune è che non vi sia uno strumento adeguato in grado di rispondere alle esigenze dell'Infermiere di Dialisi. Nonostante l'invito espresso, nessun Centro ha messo a disposizione il proprio strumento in uso per un confronto.

In merito al testing della SVC sono emerse delle criticità: alcuni infermieri l'hanno considerato troppo lungo da compilare e ridurrebbero le 86 attività accorpandole dove è possibile, altri infermieri hanno valutato alcune attività elencate di competenza medica; questo può essere riconducibile all'alta valenza discrezionale che caratterizza la professione infermieristica e all'influenza che questo esercita nell'organizzazione del contesto. Parte degli infermieri preferirebbe che il terzo grado della scheda possedesse tre livelli (7-9), benché fosse stato concepito come unico dal momento che rappresenta un Professionista di altissimo livello. Alcuni Infermieri vorrebbero una definizione più precisa dei livelli per essere più sicuri di non commettere errori valutativi. Un C.I. preferirebbe avere due SVC distinte per la valutazione continua e per l'infermiere neoassunto.

Insieme alle criticità sono emersi anche giudizi favorevoli: strumento estremamente utile poiché esplora tutti gli aspetti che condizionano il lavoro in dialisi, strumento specifico, valido a favorire l'autoriflessione e l'autovalutazione, completo e con una buona definizione delle competenze.

Alcuni limiti imposti dalla SVC sono insiti nella scelta di averla declinata direttamente da un Profilo delle Competenze che trova la sua massima espressione se lasciato nel contesto di nascita.

\section{Conclusioni}

L'obiettivo di costruire uno strumento specifico per la valutazione dell'Infermiere in Dialisi, è da considerarsi parzialmente raggiunto perché i Centri hanno definito la SVC specifica, precisa e completa seppur non scevra dall'essere migliorata.

Una validazione della SVC avrebbe necessitato di numeri maggiori e con questa riflessione vorrei stimolare la collaborazione tra gli infermieri al fine di conoscere e migliorare nuovi strumenti di lavoro.

Sarà utile proseguire il cammino professionale intrapre- so per raffinare lo strumento, soprattutto nella parte riguardante l'assegnazione del punteggio, perché diventi, attraverso rivalutazioni periodiche, un utile mezzo di valutazione e autovalutazione per il Professionista di Dialisi.

\section{Riassunto}

La valutazione delle competenze dei professionisti della salute è sicuramente un fattore strategico per l'eccellenza del Sistema Sanitario Nazionale, nonché una responsabilità alla quale il Coordinatore Infermieristico (C.I.) non dovrebbe venir meno. Obiettivo del presente lavoro è stata l'identificazione di una Scheda di Valutazione delle Competenze (SVC) quale strumento di valutazione oggettiva e stimolo all'autoriflessione e autovalutazione per l'infermiere, e per il neoassunto in dialisi.

Il progetto si è sviluppato costituendo un Profilo delle Competenze $(\mathrm{PdC})$ specifico per l'infermiere delle unità di dialisi.

Appreso che all'estero l'infermiere di dialisi possiede una specializzazione certificata e che i metodi di valutazione si basano sull'identificazione delle singole attività specifiche per ogni ruolo e l'assegnazione di un punteggio attraverso scale tipo Likert., si è proceduto all'elaborazione della SVC declinata dal PdC. La scheda è stata distribuirla in 26 Centri Dialisi di Piemonte e Valle d'Aosta attraverso il metodo di indagine 'Delphi'. Parallelamente, è stato inviato un questionario indirizzato ai C.I. per indagare il processo di valutazione presso i loro servizi. Ha aderito all'indagine il 38.5\% dei Centri: la SVC è stata testata anche su 20 infermieri; nessuno ha espresso un giudizio totalmente negativo, la SVC è stata considerata una scheda valutativa completa, in grado di favorire l'autoriflessione e l'autovalutazione, nonché un 'ottimo strumento specifico' potenziale: una effettiva validazione dello strumento necessita di un maggior numero di valutazioni e verifiche tra pari.

Parole Chiave: Autovalutazione, Competenza, Valutazione

Indirizzo degli Autori:

Luisa Napoli

Vicolo E. Fermi 1

10080 Bosconero (TO)

napoli.lu70@libero.it 


\section{Allegato}

\section{Scheda di Valutazione delle Competenze}

La SVC è organizzata in tre gradi di identificazione delle abilità dell' Infermiere - principiante, competente, esperto - suddivisi a loro volta in livelli, tre per i primi due gradi, uno per il terzo grado, determinando una totalità di sette livelli di apprendimento/ abilità. Sono stati individuati 86 elementi di attività ( distinti da un numero progressivo per una rapida identificazione ) suddivisi in 4 funzioni (assistenza, educazione, prevenzione, formazione) e in 13 sottoaree. Ad ogni elemento di attività verrà corrisposto il punteggio del livello di riferimento (da $1 \mathrm{a}$ 7); ci sarà quindi un punteggio minimo da raggiungere che cambierà a seconda che si valuti un Infermiere neoassunto ( 258 punti ), o uno che lavora da almeno un anno ( 430 punti) e un punteggio massimo rappresentato ad esempio dal raggiungimento del livello 7 per tutte le attività elencate (602 punti ).

\section{$1^{\circ}$ PRINCIPIANTE :}

Livello 1 - conosce approfonditamente la fisiopatologia del rene, conosce gli elementi teorici di base a supporto di tutte le metodiche dialitiche in uso, conosce la documentazione cartacea indispensabile a seguire le prescrizioni mediche per erogare la seduta dialitica (al termine della prima settimana di addestramento)

Livello 2 - può operare sotto la supervisione di colleghi più esperti o del responsabile su pazienti clinicamente stabili e metodiche di base poiché conosce gli aspetti pratici indispensabili all'erogazione dell'assistenza in dialisi, conosce la teoria alla base degli accessi vascolari (F.A.V. e C.V.C. ) e nella pratica la gestione del C.V.C. , conosce le principali complicanze dialitiche (ipotensione, collasso, crampi, ipertensione, aritmie, ecc.), i principali incidenti in dialisi (embolia gassosa, emolisi, rottura del filtro, coagulazione del circuito extracorporeo e crisi pirogenica) e la teoria relativa all'intervento da applicare [due pazienti cronici, metodiche HD e HFR on line, accesso vascolare C.V.C., un solo tipo di monitor (al termine della terza settimana di addestramento)]

Livello 3 - gestisce in modo autonomo la seduta dialitica sul paziente cronico (tre pazienti cronici, accesso vascolare C.V.C. e F.A.V., utilizza tutti i monitor) conosce tutti i tipi di metodiche: HD,HFR on line, AFB, HDF on line, $\mathrm{HF}$, conosce l'organizzazione della sala dialisi (approvvigionamento di farmaci, di materiale di dialisi, controllo scadenze, controllo carrelli di emergenza, turni dei pa- zienti, trasporto dei pazienti in regime di ricovero e/o ambulatoriale, documentazione cartacea, locazione e controllo degli apparecchi elettromedicali, la comunicazione tramite bacheca, la turnistica del personale), conosce il sistema informatizzato, conosce e applica l'assistenza al paziente chiamato dal Centro Trapianti renali (seduta dialitica, esami ematochimici e strumentali, bleeding), conosce gli elementi teorici di supporto al funzionamento dell'osmosi, conosce l'organizzazione relativa alla sala chirurgica di dialisi, agli ambulatori di pre-dialisi, pre-trapianto, post-trapianto, emodialisi domiciliare, dialisi peritoneale). Ricopre i tre turni ( al termine della nona settimana di addestramento)

I livelli 1-2-3 caratterizzanti il primo grado non possono non essere pienamente raggiunti dall' Infermiere neoassunto al termine del suo addestramento ( 9 settimane secondo il Protocollo interno alla S.C. ) e periodo prova di sei mesi al fine di considerare positiva la sua performance.

\section{$2^{\circ}$ COMPETENTE :}

Livello 4 - conosce le metodiche dialitiche tipiche della gestione del paziente acuto (CPFA, CVVH, CVVHD, HVHF), conosce perfettamente la gestione del sistema di purificazione dell'acqua attraverso osmosi, gestisce in modo autonomo la seduta dialitica del paziente acuto (addestramento ulteriore di due settimane) ed entra nel turno di pronta disponibilità. Procede in completa autonomia nello svolgimento di processi e situazioni complesse anche di natura non strettamente tecnicodialitica (visione completa del paziente multi patologico, capacità di prevenire complicanze a lungo termine ad es. su pazienti diabetici, supporto psicologico nell'accettazione alla malattia con la collaborazione di personale specializzato, corretto indirizzo nello svolgimento di pratiche sociali come ad es. invalidità, esenzioni, pensioni, accompagnamenti)

Livello 5 - gestisce in completa autonomia la risoluzione di situazioni imprevedibili attraverso un approccio innovativo (si avvale dell'uso dell'EBN/M, coinvolge i colleghi, stimola il lavoro in equipe ed il miglioramento continuo)

Livello 6 - forma il personale neoassunto

I livelli 4-5 del secondo grado devono necessariamente caratterizzare gli Infermieri in servizio da più di un anno, ed il livello 6 è raggiungibile attraverso caratteristiche molto personali e attitudini specifiche all'insegnamento. 


\section{$3^{\circ}$ ESPERTO:}

Livello 7 - ha conoscenza completa di tutti gli aspetti relativi alla S.C. di Nefrologia e Dialisi , siano essi strutturali od organizzativi. Svolge un'assistenza completa e di alta qualità al paziente uremico conoscendo in modo approfondito la clinica, le complicanze di natura psico-sociale, le tecniche e le complicanze dialitiche, ha capacità di stimolare il lavoro multi professionale, ha interesse a stimolare e sviluppare lavori all'interno del gruppo a supporto di nuovi argomenti secondo la necessità della popolazione in cura( paziente straniero con difficoltà legate alla lingua, paziente diabetico con complicanze di ulcere, programmi di educazione al paziente pre dialitico, al paziente trapiantato, gestione degli accessi vascolari, ecc.), è responsabile, attraverso la delega da parte del Coordinatore, di specifiche aree allinterno della S.S. (emodialisi domiciliare, dialisi peritoneale, trapianto renale).

Il livello 7 è proprio degli operatori che posseggono attitudini, capacità, motivazioni e valori ad elevato impatto sull'organizzazione. Sono Infermieri che si contraddistinguono per il loro impegno costante e la volontà continua di intraprendere particolari percorsi formativi.

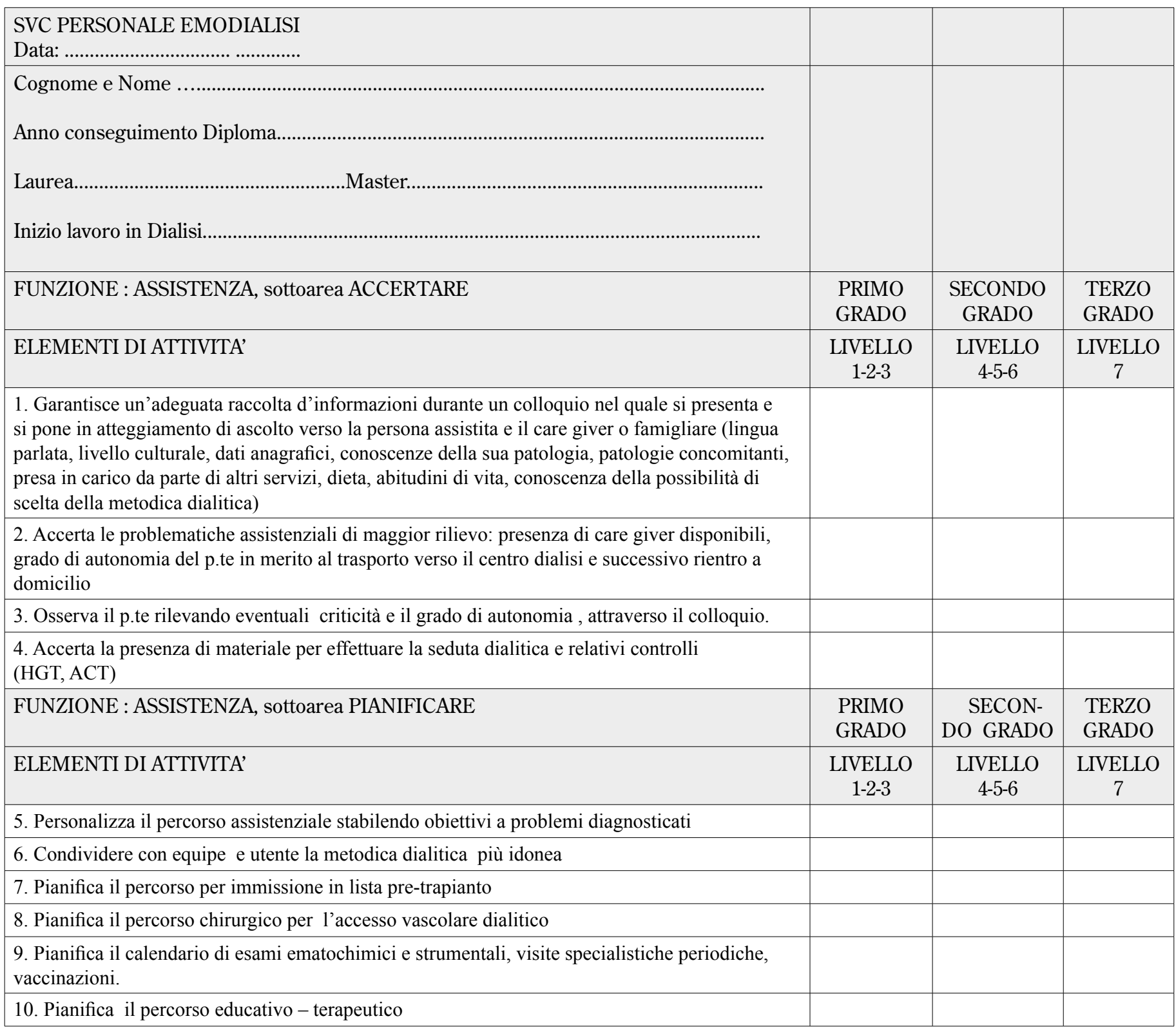




\begin{tabular}{|c|c|c|c|}
\hline \multicolumn{4}{|l|}{$\begin{array}{l}\text { 11. Pianifica con personale referente (servizio economale e ditte in service) la fornitura } \\
\text { di materiale dialitico }\end{array}$} \\
\hline $\begin{array}{l}\text { 12. Pianifica i vari percorsi assistenziali, con approccio multidisciplinare/multiprofessionale, intra } \\
\text { ed extra ospedalieri per favorire la continuità assistenziale [ricovero, RSA, CT, ricovero presso } \\
\text { altre Strutture Complesse }(\mathrm{SC}) \text { ] }\end{array}$ & & & \\
\hline 13. Pianifica il periodo di “assenza” dal centro di riferimento (vacanze, interventi terapeutici) & & & \\
\hline 14. Pianifica il programma dialitico settimanale di tutti i pazienti (cronici e acuti) & & & \\
\hline FUNZIONE : ASSISTENZA, sottoarea ATTUARE & $\begin{array}{l}\text { PRIMO } \\
\text { GRADO }\end{array}$ & $\begin{array}{l}\text { SECONDO } \\
\text { GRADO }\end{array}$ & $\begin{array}{l}\text { TERZO } \\
\text { GRADO }\end{array}$ \\
\hline ELEMENTI DI ATTIVITA' & $\begin{array}{l}\text { LIVELLO } \\
1-2-3\end{array}$ & $\begin{array}{l}\text { LIVELLO } \\
4-5-6\end{array}$ & $\begin{array}{l}\text { LIVELLO } \\
\quad 7\end{array}$ \\
\hline $\begin{array}{l}\text { 15. Attua interventi clinico assistenziali in base all'intensità di cura e alla complessità } \\
\text { assistenziale (comorbilità con insufficienza renale: diabete, cardiopatia, vasculopatia, } \\
\text { patologia psichiatrica senilità e multietnicità) }\end{array}$ & & & \\
\hline $\begin{array}{l}\text { 16. Attua l'accoglienza dell'utente e del care giver attraverso colloquio e materiale informativo } \\
\text { (opuscoli, diapositive, riunioni multidisciplinari pre dialisi) }\end{array}$ & & & \\
\hline $\begin{array}{l}\text { 17. Gestisce la documentazione clinica e assistenziale attraverso cartella clinica cartacea ed } \\
\text { informatizzata (Infogramma) e diario cartaceo della seduta dialitica }\end{array}$ & & & \\
\hline 18. Applica procedure, protocolli e istruzioni operative al trattamento dialitico e ai PDTA & & & \\
\hline $\begin{array}{l}\text { 19. Attua il calendario personalizzato degli accertamenti clinico diagnostici } \\
\text { (settimanale, mensile, annuale e personalizzato in caso di criticità) }\end{array}$ & & & \\
\hline $\begin{array}{l}\text { 20. Applica procedure di approvvigionamento materiale sanitario (Economato, } \\
\text { Provveditorato farmaceutico) }\end{array}$ & & & \\
\hline $\begin{array}{l}\text { 21. Attua contatti con vari professionisti specialisti (dietista, assistente sociale, psicologo/ } \\
\text { psichiatra etc.) }\end{array}$ & & & \\
\hline FUNZIONE : ASSISTENZA, sottoarea VALUTARE & $\begin{array}{l}\text { PRIMO } \\
\text { GRADO }\end{array}$ & $\begin{array}{l}\text { SECONDO } \\
\text { GRADO }\end{array}$ & $\begin{array}{l}\text { TERZO } \\
\text { GRADO }\end{array}$ \\
\hline ELEMENTI DI ATTIVITA' & $\begin{array}{l}\text { LIVELLO } \\
1-2-3\end{array}$ & $\begin{array}{l}\text { LIVELLO } \\
4-5-6\end{array}$ & $\begin{array}{l}\text { LIVELLO } \\
\quad 7\end{array}$ \\
\hline 22. Valuta l'efficacia del piano assistenziale pianificato & & & \\
\hline $\begin{array}{l}\text { 23. Valuta complicanze terapia farmacologica (sovradosaggio antinfiammatori, anticoagulanti e } \\
\text { antibiotici ad escrezione renale, allergie e intolleranze, variazione dell'emivita del farmaco } \\
\text { in corso di trattamento dialitico ) }\end{array}$ & & & \\
\hline $\begin{array}{l}\text { 24. Valuta le informazioni in possesso dell'assistito e del care giver e loro grado di comprensione } \\
\text { (osservazione diretta, colloquio ) }\end{array}$ & & & \\
\hline $\begin{array}{l}\text { 25. Valuta aderenza terapeutica, dietetica e stile di vita attraverso controlli strumentali, } \\
\text { ematochimici. }\end{array}$ & & & \\
\hline 26. Valuta la corretta compilazione schede dialitiche & & & \\
\hline 27. Valuta la corretta "dose" dialitica (KT/V) & & & \\
\hline FUNZIONE : ASSISTENZA, sottoarea GESTIRE i PDTA e RISCHIO BIOLOGICO & $\begin{array}{l}\text { PRIMO } \\
\text { GRADO }\end{array}$ & $\begin{array}{l}\text { SECONDO } \\
\text { GRADO }\end{array}$ & $\begin{array}{l}\text { TERZO } \\
\text { GRADO }\end{array}$ \\
\hline ELEMENTI DI ATTIVITA' & $\begin{array}{l}\text { LIVELLO } \\
1-2-3\end{array}$ & $\begin{array}{l}\text { LIVELLO } \\
4-5-6\end{array}$ & $\begin{array}{l}\text { LIVELLO } \\
\quad 7\end{array}$ \\
\hline 28. Gestisce il percorso assistenziale del paziente late referral (cronico in urgenza dialitica) & & & \\
\hline 29. Gestisce il percorso assistenziale del nuovo ingresso paziente in terapia sostitutiva & & & \\
\hline $\begin{array}{l}\text { 30. Gestisce il percorso assistenziale e la preparazione del paziente dialitico a interventi e } \\
\text { procedure diagnostico/terapeutiche }\end{array}$ & & & \\
\hline $\begin{array}{l}\text { 31. Gestisce il percorso assistenziale con altre SC (urologia, cardiologia, chirurgia vascolare, } \\
\text { centro trapianti, etc.) }\end{array}$ & & & \\
\hline
\end{tabular}


32. Gestisce l'accesso vascolare dialitico (utilizzo precoce, tamponamento, programma di prevenzione)

33. Sorveglia il corretto approvvigionamento e utilizzo del materiale dialitico secondo prescrizione

34. Sorveglia quotidianamente la qualità dell'acqua osmotizzata

35. Sorveglia ed esegue correttamente le procedure di decontaminazione, pulizia e disinfezione di apparecchiature di dialisi, sanitarie e locali

36. Identifica i rischi clinici maggiori : biologico, clinico, infettivo, puntura accidentale 37. Sorveglia ed esegue il corretto smaltimento rifiuti ospedalieri (ematici, citostatici, taglienti etc.)

38. Gestisce il corretto percorso del farmaco (protocollo aziendale e di SC, KCL, citostatici, immunoglobuline, terapia EV corticosteroidea)

39. Sorveglia ed attua il corretto utilizzo dei DPI

FUNZIONE : ASSISTENZA, sottoarea GESTIRE la seduta di Dialisi

\section{ELEMENTI DI ATTIVITA'}

40. Controlla il materiale dialitico (in conformità alla prescrizione - scheda dialisi)

41. Prepara il monitor dialitico

42. Accompagna l'utente alla propria unità dialitica in collaborazione con OSS

43. Documenta i parametri vitali prima di iniziare la seduta ed eventuali criticità intercorse nel periodo interdialitico

44. Imposta i parametri sul monitor (tempo, disidratazione, eparina etc.)

45. Pratica le manovre di "attacco" dell'utente al circuito extracorporeo (FAV, protesi , CVC)

46. Documenta sistematicamente, ogni ora, i parametri vitali sulla scheda dialitica e intervenire tempestivamente in caso di instabilità emodinamica (ipotensione, crampi, emolisi etc)

47. Somministra terapie intradialitiche secondo prescrizione

48. Pratica le manovre di "stacco" dell'utente dal circuito extracorporeo (tamponamento FAV, chiusura $\mathrm{CVC}$, etc.)

49. Congeda l'utente e accerta l'effettivo rientro presso il proprio domicilio e/o reparto di degenza

50. Gestisce l'unità dialitica (detersione, disinfezione monitor, smaltimento rifiuti, detersione, sanitizzazione, riordino materiale )

51. Ripristina il materiale dialitico per il trattamento dell' utente successivo

FUNZIONE : ASSISTENZA, sottoarea GESTIRE le complicanze, la richiesta di soccorso

\section{ELEMENTI DI ATTIVITA'}

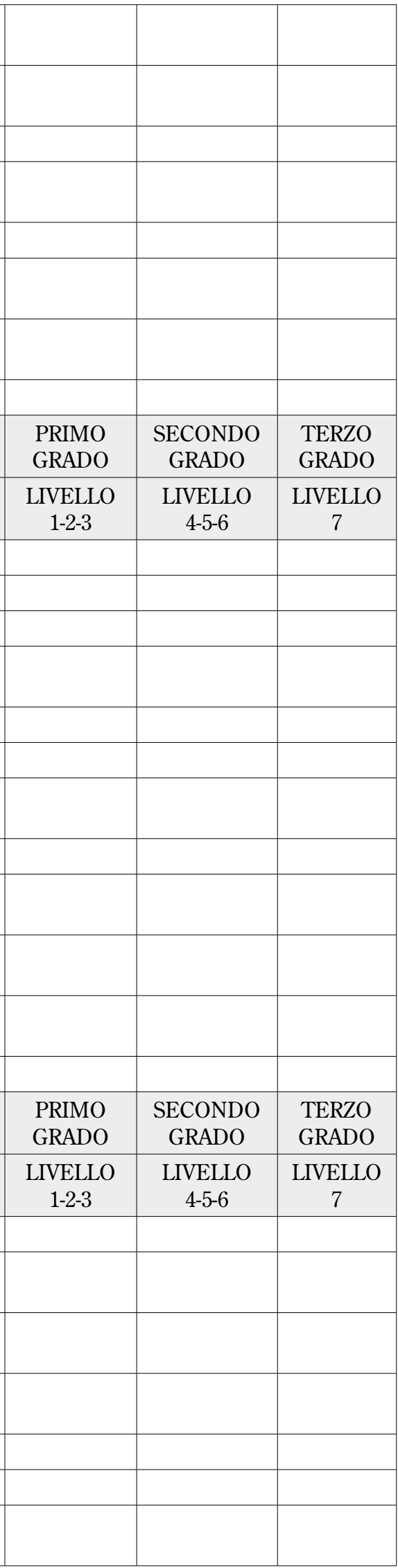

52. Identifica segni e sintomi rilevanti (dispnea, tremori, rigidità muscolare, ipertermia)

53. Collabora con il medico nell'organizzare l'intervento appropriato (dialisi supplementare, terapia, esami colturali ed ematochimici, monitoraggio segni vitali etc.)

54. Interviene tempestivamente durante il trattamento dialitico nell'insorgere delle complicanze (ipo o ipertensione, emolisi, ipo o iperglicemie, emorragia, arresto cardiorespiratorio)

55. Interviene tempestivamente in caso di reazioni allergiche durante la somministrazione di terapie particolari (antibiotici etc.)

56. Conosce la tecnica di BLS-D e i presidi per attuarlo

57. Conosce l'esistenza del Servizio "SEI" e sa attivarlo

58. Riconosce tempestivamente i sintomi riferiti telefonicamente dall'utente (sovraccarico idrico, disidratazione, ipertermia) 


\begin{tabular}{|c|c|c|c|}
\hline $\begin{array}{l}\text { 59. Indirizza l'utente che chiama telefonicamente, ove necessario, alla struttura sanitaria più } \\
\text { idonea (DEA, centro dialisi) }\end{array}$ & & & \\
\hline $\begin{array}{l}\text { 60. Riconosce eventuali problemi legati alla dialisi domiciliare (utilizzo di apparecchiature } \\
\text { dialitiche) }\end{array}$ & & & \\
\hline 61. Applica il protocollo aziendale di “ maxi emergenza” per quanto di competenza & & & \\
\hline 62. Collabora con altri centri dialisi in caso di maxiemergenza & & & \\
\hline FUNZIONE : ASSISTENZA, sottoarea GESTIRE il disagio psicologico del paziente & $\begin{array}{l}\text { PRIMO } \\
\text { GRADO }\end{array}$ & $\begin{array}{l}\text { SECONDO } \\
\text { GRADO }\end{array}$ & $\begin{array}{l}\text { TERZO } \\
\text { GRADO }\end{array}$ \\
\hline ELEMENTI DI ATTIVITA' & $\begin{array}{l}\text { LIVELLO } \\
1-2-3\end{array}$ & $\begin{array}{l}\text { LIVELLO } \\
4-5-6\end{array}$ & $\begin{array}{l}\text { LIVELLO } \\
\quad 7\end{array}$ \\
\hline $\begin{array}{l}\text { 63. Personalizza gli interventi a seconda dei bisogni dei pazienti avvalendosi di figure } \\
\text { multidisciplinari (medici, infermieri, psicologi, psichiatri, dietista, ass. sociale) fornendo } \\
\text { informazioni relative a problemi psicologici, clinici, sessuali, socio lavorativi, alimentari, } \\
\text { modificazioni dell'immagine di sé }\end{array}$ & & & \\
\hline $\begin{array}{l}\text { 64. Gestisce le problematiche del cambiamento di vita (inizio trattamento sostitutivo, rientro } \\
\text { in dialisi dopo trapianto) }\end{array}$ & & & \\
\hline $\begin{array}{l}\text { 65. Accoglie il "nuovo" paziente cronico all'inizio del suo percorso dialitico con disponibilità } \\
\text { e linguaggio appropriati atti a diminuire l'ansia }\end{array}$ & & & \\
\hline $\begin{array}{l}\text { 66. Gestisce improvvisi episodi di "rifiuto" della malattia cronica (mancata aderenza alla terap } \\
\text { dialitica, farmacologica, ed elaborazione del lutto di un altro paziente) }\end{array}$ & & & \\
\hline FUNZIONE : ASSISTENZA, sottoarea GESTIRE il disagio psicologico del personale & $\begin{array}{l}\text { PRIMO } \\
\text { GRADO }\end{array}$ & $\begin{array}{c}\text { SECONDO } \\
\text { GRADO }\end{array}$ & $\begin{array}{c}\text { TERZO } \\
\text { GRADO }\end{array}$ \\
\hline ELEMENTI DI ATTIVITA' & $\begin{array}{l}\text { LIVELLO } \\
1-2-3\end{array}$ & $\begin{array}{l}\text { LIVELLO } \\
4-5-6\end{array}$ & $\begin{array}{l}\text { LIVELLO } \\
\quad 7\end{array}$ \\
\hline $\begin{array}{l}\text { 67. Favorisce l'inserimento in equipe del neo assunto allo scopo di superare il suo senso } \\
\text { di inadeguatezza (accoglienza e protocollo di addestramento specifico) }\end{array}$ & & & \\
\hline $\begin{array}{l}\text { 68. Gestisce il proprio stato emotivo nella relazione con il paziente cronico, l'incidente critico } \\
\text { e l'elaborazione del lutto attraverso programmi volti alla gestione del vissuto emotivo } \\
\text { (eventi formativi, debriefing con l'equipe dopo eventi critici ) }\end{array}$ & & & \\
\hline 69. Riconosce comportamenti e reazioni a rischio di burn out & & & \\
\hline 70. Favorisce l'approccio agli strumenti tecnologici (apparecchiature dialitiche) & & & \\
\hline $\begin{array}{l}\text { 71. Gestisce il conflitto all'interno dell'equipe derivante dalla tipologia di lavoro in ambiente } \\
\text { "chiuso" }\end{array}$ & & & \\
\hline $\begin{array}{l}\text { FUNZIONE : EDUCAZIONE, sottoarea REALIZZAZIONE di interventi di educazione } \\
\text { terapeutica }\end{array}$ & $\begin{array}{l}\text { PRIMO } \\
\text { GRADO }\end{array}$ & $\begin{array}{l}\text { SECONDO } \\
\text { GRADO }\end{array}$ & $\begin{array}{l}\text { TERZO } \\
\text { GRADO }\end{array}$ \\
\hline ELEMENTI DI ATTIVITA' & $\begin{array}{l}\text { LIVELLO } \\
1-2-3\end{array}$ & $\begin{array}{l}\text { LIVELLO } \\
4-5-6\end{array}$ & $\begin{array}{l}\text { LIVELLO } \\
\quad 7\end{array}$ \\
\hline $\begin{array}{l}\text { 72. Attua interventi informativi/educativi al nuovo utente, ai famigliari e care giver } \\
\text { (dieta, terapia, igiene, stile di vita) }\end{array}$ & & & \\
\hline $\begin{array}{l}\text { 73. Educa, in modo personalizzato, il paziente in trattamento sostitutivo: } \\
\text { - gestione dell'accesso dialitico } \\
\text { - terapia dietetica (fosforo, potassio, liquidi, proteine, glucidi etc) } \\
\text { - terapia farmacologica (eritropoietina, chelanti del fosforo, ipotensivi, corticosteroidi, } \\
\text { immunosoppressori etc.) }\end{array}$ & & & \\
\hline $\begin{array}{l}\text { 74. Educa l'utente, famigliari e care giver a riconoscere tempestivamente segni e sintomi } \\
\text { di alterazioni dello stato di salute richiedendo l'intervento opportuno }\end{array}$ & & & \\
\hline 75. Individua ostacoli all'apprendimento (livello culturale, lingua, religione etc) & & & \\
\hline 76. Educa il paziente a raggiungere un buon livello di autonomia e autogestione & & & \\
\hline $\begin{array}{l}\text { 77. Verifica il grado di apprendimento rispetto a informazioni date e/o comportamenti appresi } \\
\text { (aderenza a controlli e visite specialistiche, alla terapia e stili di vita) }\end{array}$ & & & \\
\hline
\end{tabular}




\begin{tabular}{|c|c|c|c|}
\hline FUNZIONE : PREVENZIONE, sottoarea REALIZZAZIONE di interventi di prevenzione & $\begin{array}{l}\text { PRIMO } \\
\text { GRADO }\end{array}$ & $\begin{array}{l}\text { SECONDO } \\
\text { GRADO }\end{array}$ & $\begin{array}{r}\text { TERZO } \\
\text { GRADO }\end{array}$ \\
\hline ELEMENTI DI ATTIVITA' & $\begin{array}{l}\text { LIVELLO } \\
1-2-3\end{array}$ & $\begin{array}{l}\text { LIVELLO } \\
4-5-6\end{array}$ & $\begin{array}{l}\text { LIVELLO } \\
\quad 7 \\
\end{array}$ \\
\hline \multicolumn{4}{|l|}{$\begin{array}{l}\text { 78. Educa il paziente portatore di FAV a sorvegliarla, gestirla, preservarla (arrossamenti, } \\
\text { sanguinamenti, trill, compressioni ) }\end{array}$} \\
\hline \multicolumn{4}{|l|}{$\begin{array}{l}\text { 79. Monitorizza i markers infettivologici ed organizza, quando previste, eventuali vaccinazioni } \\
\text { (ematochimici a cadenza periodica) }\end{array}$} \\
\hline \multicolumn{4}{|l|}{$\begin{array}{l}\text { 80. Sensibilizza il paziente ad aderire ai programmi di prevenzione istituzionali (oncologico, } \\
\text { cardiovascolare, fumo) e specifici (rischio oncologico e infettivo aumentato } \\
\text { da immunosoppressione) }\end{array}$} \\
\hline \multicolumn{4}{|l|}{$\begin{array}{l}\text { 81. Collabora alle campagne di prevenzione (giornata mondiale del rene, diabete, ipertensione, } \\
\text { trapianto) e divulgare informazioni tramite depliants }\end{array}$} \\
\hline FUNZIONE : FORMAZIONE, sottoarea REALIZZAZIONE di programmi di formazione & $\begin{array}{l}\text { PRIMO } \\
\text { GRADO }\end{array}$ & $\begin{array}{l}\text { SECONDO } \\
\text { GRADO }\end{array}$ & $\begin{array}{l}\text { TERZO } \\
\text { GRADO }\end{array}$ \\
\hline ELEMENTI DI ATTIVITA' & $\begin{array}{l}\text { LIVELLO } \\
1-2-3\end{array}$ & $\begin{array}{l}\text { LIVELLO } \\
4-5-6\end{array}$ & $\begin{array}{l}\text { LIVELLO } \\
\quad 7\end{array}$ \\
\hline \multicolumn{4}{|l|}{ 82. Rileva i propri bisogni formativi e attua un programma di autoaggiornamento } \\
\hline \multicolumn{4}{|l|}{ 83. Uniforma le proprie conoscenze e competenze organizzando e partecipando a corsi mirati } \\
\hline \multicolumn{4}{|l|}{ 84. Condivide le conoscenze apprese (EBM/N, congressi) } \\
\hline \multicolumn{4}{|l|}{$\begin{array}{l}\text { 85. Collabora ad elaborare materiale didattico aggiornabile per il neoassunto e libero } \\
\text { professionista per apprendere le procedure dialitiche ( HDB, AFB, HFR, HDF on line } \\
\text { ed emodialitiche intermittenti ) }\end{array}$} \\
\hline \multicolumn{4}{|l|}{$\begin{array}{l}\text { 86. Collabora nell'organizzare corsi formativi/informativi teorico/pratici sulle metodiche } \\
\text { dialitiche ad altre SC di "area critica" }\end{array}$} \\
\hline \multicolumn{4}{|l|}{ PUNTEGGIO TOTALIZZATO } \\
\hline PUNTEGGIO MASSIMO RAGGIUNGIBILE & & & 609 \\
\hline LIMITE INFERIORE DI PUNTEGGIO PER IL NEOASSUNTO & & & 258 \\
\hline $\begin{array}{l}\text { LIMITE INFERIORE DI PUNTEGGIO PER L'INFERMIERE CHE LAVORA IN DIALISI DA } \\
\text { PIU' DI UN ANNO }\end{array}$ & & & 430 \\
\hline
\end{tabular}

Aree da potenziare attraverso l'impegno, l'addestramento e/o eventi formativi :........

Firma dell' Infermiere
Firma del Coordinatore Infermieristico 


\section{Bibliografia}

1. DM 14 Settembre $1994 \mathrm{n}^{\circ} 739$ "Regolamento concernente l'individuazione della figura e del relativo profilo professionale" IPASVI 10.01.2009 Delibera $\mathrm{n}^{\circ} 1 / 09$ Legge 26 Febbraio $1999 \mathrm{n}^{\circ} 42$ "Disposizioni in materia di professioni sanitarie” D.P.R. 14 Marzo 1974 n 225 (abrogata dalla L. 42/99, ad esclusione del titolo quinto mansionario dell'infermiere generico) Legge 10 Agosto 2000 $\mathrm{n}^{\circ} 251$ "Disciplina delle professioni sanitarie infermieristiche, tecniche, della riabilitazione della prevenzione nonché della professione ostetrica".

2. K.M. Wiig. Societal knowledge management in the globalised economy”. International Journal of Advanced Media

3. Rivista. Linfermiere notiziario aggiornamenti professionali 2007; 4: 6-7.

4. Accordo per il lavoro del 24 Settembre 1996 tra il Governo e le parti sociali Legge 24 Giugno 1997 n $^{\circ} 196$ "Norme in materia di promozione dell'occupazione" Legge 15 Marzo 1997 n 59 "Delega al Governo per il conferimento di funzioni e compiti alle regioni ed enti locali, per la riforma della Pubblica Amministrazione e per la semplificazione amministrativa" D.Lgs. 502/92e successive modifiche sono il quadro di riferimento: l'art. 8 sulla "Disciplina dei rapporti per l'erogazione di prestazioni assistenziali”, l'art. 10 sul "Controllo di qualità", l'art. 14 sui "Diritti dei cittadini”. http://bancadati.italialavoro.it Legge $1^{\circ}$ Febbraio $2006 \mathrm{n}^{\circ} 43$ "Disposizioni in materia di professioni sanitarie infermieristiche, ostetrica, riabilitative, tecnicosanitarie e della prevenzione e delega al Governo per l'istituzione dei relativi ordini professionali”.

5. Allegato 1 Scheda di Valutazione delle Competenze

6. B.F. Prowant. Focus on issues related to Nephrology Nursing Certification: how certification examinations are developed. Nephrol Nur J 2008; 35(6).

7. Parker J. Certified Dialysis Nurse (CDN) examination: exploring the issues. Nephrol Nurs J 2000; 27 (4): 351-419. Comment in: Nephrol Nurs J 2000; 27(5): 456. PMID: 11276625.

8. Simard JC, Zarifian A. Certified Dialysis Nurse (CDN) examination: pros and cons. Intermountain Health Care, Salt Lake City, UT, USA. Nephrol Nurs J 2000; 27 (4): 4423. Comment in: Nephrol Nurs J 2000; 27(5): 456. PMID: 11276635.

9. Thomas N. Clinical standards and guidelines. EDTNA ERCA J 2005; 312): 60-2. SW Thames Renal Transplantation Unit, St. Helier, Carshalton, Surrey, UK. thehorseshoe@btopenworld.com PMID: 16180548
10. A Zampieron, P.Ormandy. Comparison of Nephrology Nursing interventions across five Europan Countries. J Renal Care 2009.

11. Allen P, Lauchner K, Bridges R.A, Francis-Johnson P, McBride S.G, Olivarez A. Evaluating Continuing Competency: A Challenge for Nursing. J Contin Educ Nurs 2008; 39(2): 81-5.

12. McCarthy G, Fitzpatrick J.J. Development of a Competency Framework for Nurse Managers in Ireland. J Contin Educ Nurs 2009; 40(8): 346-50.

13. Bonner B.A, Greenwood J. The acquisition and exercise of nephrology nursing expertise: a grounded theory study" . Renal Nursing. Acquisition and exercise of nephrology nursing. 2006 Blackwell Publishing Ltd, J Clinical Nursing, 15: 480-9.

14. Zampieron A, Elseviers M, Ormandy P, Vlaminck H, De Vos JY, Kafkia T, Lindley E, Harrington M. Development of indicators to measure European variation of nursing activities. EDTNA ERCA J 2006; 32(1): 14-9.

15. McCann M, Sedgewick J. Royal. The European Post-Basic Core Curriculum (PBCC) 2nd Edition for Nephrology Nursing: Progress Update. College of Surgeons, Ireland. EDTNA ERCA J 2005; 31(2):115-7.

16. Fuchs S, Thomas N. The European Basic Core Curriculum for Nephrology Nursing, the process of writing, publishing and disseminating. EDTNA ERCA J 2003; 29(4):178-80. St. Helier Hospital, Renal Unit, Carshalton, Surrey, UK .

17. Meretoja R, Isoaho H, Leino-Kilpi H. Nurse competence scale: development and psychometric testing. J Adv Nurs 2004; 47 (2): 124-33. Department of Nursing Science, University of Turku and Corporate Headquarters, Hospital District of Helsinki and Uusimaa, Helsinki, Finland. PMID: 15196186 [PubMed - indexed for MEDLINE]

18. Spencer L., Spencer M. Competenza nel lavoro. Milano: Franco Angeli, 1995 (Originale, 1993). A. Saggin. Le competenze necessarie per lavorare nelle organizzazioni sanitarie con soddisfazione: competenze per il benessere individuale e competenze per lavorare con gli altri. SDA Bocconi Milano. Relazione Convegno Empoli Novembre 2003 W. Levati, M.V. Saraò. "Il modello delle competenze”. FrancoAngeli. IV Ed. 2003.

19. Bernard A. Gestire la motivazione per migliorare la performance, in: Carretta, Dalziel, Mitrani, Dalle risorse umane alle competenze, Milano: Hay Group, 1992, pag. 154.

20. Bolognini M. Democrazia elettronica, metodo Delphi e politiche pubbliche. Roma: Ed Carocci, 2001; 81-95. 